\title{
Optimization of the contents of Arabic gum, xanthan gum and orange oil affecting turbidity, average particle size, polydispersity index and density in orange beverage emulsion
}

\begin{abstract}
This paper focuses on the development of an effective methodology to determine the optimum levels of three independent variables leading to (a) maximize turbidity, (b) minimize polydispersity index (PDI) and (c) obtain the target value for average particle size and density of orange beverage emulsion. A three-factor central composite design (CCD) was employed to determine the effect of Arabic gum content $(7-13 \% \mathrm{w} / \mathrm{w})$, xanthan gum content $(0.1-0.3 \% \mathrm{w} / \mathrm{w})$ and orange oil content $(6-10 \% \mathrm{w} / \mathrm{w})$. The emulsion properties studied as response variables were: turbidity (Y1), average particle size (Y2), PDI (Y3) and density (Y4). The response surface analysis was carried out to create efficient empirical models for predicting the changes of response variables. In general, analysis of variance (ANOVA) showed high coefficients of determination values (R2) in the range of $0.922-0.975$ for the response surface models, thus ensuring a satisfactory adjustment of the polynomial regression models with the experimental data. The results of regression analysis indicated that more than $92 \%$ the response variation could be explained by the models. The results also indicated that the linear term of xanthan gum was the most significant $(p<0.05)$ variable affecting the overall responses. The multiple optimization results showed that the overall optimum region with high total desirability $(\mathrm{D}=0.92)$ was found to be at the combined level of $13.88 \% \mathrm{w} / \mathrm{w}$ Arabic gum content, $0.27 \% \mathrm{w} / \mathrm{w}$ xanthan gum content and $11.27 \% \mathrm{w} / \mathrm{w}$ orange oil content. Under the optimum condition, the corresponding predicted response values for turbidity, average particle size, PDI and density of the desirable orange beverage emulsion were 129.55, 988, 0.261 and 1.03, respectively. For validation of the models, the experimental values were compared with predicted values to check the adequacy of the models. The experimental values were found to be in agreement with those predicted, thus indicating suitability of the models employed using response surface methodology (RSM) for optimizing the physical properties of the orange beverage emulsion.
\end{abstract}

Keyword: Orange beverage emulsion, Turbidity, Average particle size, Polydispersity index, Central composite design, Multiple optimization, Validation, Response surface methodology 